\title{
Effects of physical activity and exercise on the cognitive function of patients with Alzheimer disease: a meta-analysis
}

\author{
Rui-xia Jia ${ }^{\dagger}$, Jing-hong Liang ${ }^{\dagger}$, Yong $\mathrm{Xu}^{*}$ and Ying-quan Wang
}

\begin{abstract}
Background: Alzheimer's disease (AD), as the most common cause of dementia, brings huge economic burden for patients and social health care systems, which motivates researchers to study multiple protective factors, among which physical activity and exercise have been proven to be both effective and economically feasible.

Methods: A systematic literature search was performed for eligible studies published up to November 1st 2018 on three international databases (PubMed, Cochrane Library, and Embase) and two Chinese databases (Wanfang Data, China National Knowledge Infrastructure). All analyses were conducted using Stata 14.0. Due to heterogeneity between studies, a random-effects model was used for this meta-analysis. Meta-analysis was used to explore if physical activity and exercise can exert positive effects on cognition of elderly with $A D$ and subgroup analyses were conducted to find out if there are dose-response effects.

Results: A total of 13 randomized controlled trials were included with a sample size of 673 subjects diagnosed with AD. Intervention groups showed a statistically significant improvement in cognition of included subjects measured by the MMSE score (SMD = 1.12 Cl:0.66 1.59) compared to the control groups. Subgroup analyses showed different amounts of physical activity and exercise can generate different effects.

Conclusions: As one of few meta-analyses comparing different quantities of physical activity and exercise interventions for $A D$ in details, our study suggests that physical activity and exercise can improve cognition of older adults with AD. While the concomitant effects on cognition functions of high frequency interventions was not greater than that of low frequency interventions, the threshold remains to be settled. However, more RCTs with rigorous study design are needed to support our findings.
\end{abstract}

Keywords: Physical activity, Exercise, Alzheimer's disease, cognition, Meta-analysis, Elderly, Older adults

\section{Background}

According to World Alzheimer Report 2016, about 47 million people struggled with dementia worldwide. This number was predicted to increase to more than 131 million by 2050 , as the size and proportion of population age 65 and older continue to escalate across the world. There is also huge health care cost caused by dementia. The total estimated cost of dementia was US $\$ 818$ billion

\footnotetext{
* Correspondence: 838202104@qq.com

${ }^{+}$Ruixia Jia and Jinghong Liang contributed equally to the work presented here and therefore should be considered equivalent authors.

Department of Social Medicine, Jiangsu Key Laboratory of Preventive and Translational Medicine for Geriatric Diseases, School of Public Health, Medical College of Soochow University, No.199 Ren Ai Road, Suzhou, People's Republic of China215123
}

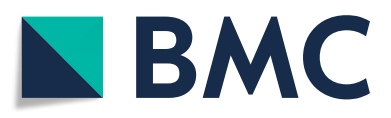

(c) The Author(s). 2019 Open Access This article is distributed under the terms of the Creative Commons Attribution 4.0 International License (http://creativecommons.org/licenses/by/4.0/), which permits unrestricted use, distribution, and reproduction in any medium, provided you give appropriate credit to the original author(s) and the source, provide a link to the Creative Commons license, and indicate if changes were made. The Creative Commons Public Domain Dedication waiver (http://creativecommons.org/publicdomain/zero/1.0/) applies to the data made available in this article, unless otherwise stated. worldwide, which is set to rise as the number of people with dementia increases [1]. There are mainly 8 causes of dementia including Alzheimer's disease(AD), Vascular dementia, Dementia with Lewy bodies, Mixed dementia, Frontotemporal lobar degeneration, Parkinson's disease dementia, Creutzfeldt-Jakob disease and Normal pressure hydrocephalus, among which $\mathrm{AD}$ is the most common cause of dementia and accounts for about 60 to $80 \%$ of cases [2].

\section{What is AD: definition, symptoms and risk factors}

$\mathrm{AD}$ is a chronic neurodegenerative disorder starting and developing insidiously, whose typical primary groups of symptoms are: (1) cognitive dysfunction; (2) psychiatric 
symptoms and behavioral disturbances; (3) difficulties with performing activities of daily living. These symptoms progress from mild memory loss to very severe dementia [3]. With the disease deteriorating, patients would lose their bodily functions and life finally. Studies show that the typical life expectancy following diagnosis is three to nine years despite of various speed of progression on different individuals $[4,5]$. AD is a devastating disease both expensive in economy and painful in emotion for patients and their families, yet few final conclusion has yet been reached on the precise biological changes that cause $\mathrm{AD}$, why its progressions vary among different patients, and how to prevent, slow or stop it [2].

The heavy damage to the society, patients and their families caused by AD motivates researchers in different fields to study risk factors and possible ways to conduct prevention and intervention against AD. For example, Miia Kivipelto et al [6] reported advanced age, family history, apolipoprotein E (apoE) $\varepsilon 4$ allele, physical inactivity, high dietary fat intake, alcohol drinking and smoking were risk factors for AD. Cedazo-Minguez A [7] had similar opinion that risk factors for AD included advanced age, family history of dementia, low educational accomplishment and presence of the $\varepsilon 4$ isoform of the apolipoprotein $\mathrm{E}$ (apoE). Rolland Y et al [8] hold that modifiable lifestyle factors such as inactivity may affect the development and progression of AD. Fratiglioni L et al [9] reported that three lifestyle factors can slow the rate of cognitive decline and preventing dementia: a socially integrated network, cognitive leisure activity, and regular physical activity. There are studies suggesting physical activity has the most support as protective against the deleterious effects of age on health and cognition $[10,11]$. Since physical activity and exercise are appealing low-cost and low-risk alternative treatment [8], more and more trails have been conducted to examine the effects of physical activity and exercise on the cognition of patients with AD.

\section{Effects of physical activity and exercise}

Recently, results from randomized controlled trails (RCTs) have suggested that people should adopt physical activity and exercise to alleviate the negative impact of aging on their cognitive function. A randomized trail conducted by Lautenschlage NT [12] in 2008 showed physical activity and exercise may slow down cognitive decline, which is in agreement with Kramer AF [13]. Heyn P et al [14] reported physical activity and exercise had positive effects on cognition among those with cognitive decline in a meta-analysis.

Evidence from all kinds of trails suggests that physical activity and exercise can to some extent improve cognition performance among patients with cognitive impairment, yet it is still unclear which combinations of frequency, intensity, time, and type of exercise can exert a better effect on improving cognition of older adults diagnosed with $\mathrm{AD}$.

To find the optimal intervention way, it is urgent to answer how to conduct physical activity and exercise RCTs to improve cognition in terms of the amount of physical activity and exercise. This meta-analysis was conducted to study the dose-response effects of physical activity on cognition of patients with $\mathrm{AD}$ as a need-to-be settled question. The findings should provide bases for establishing guidelines and recommendations for future physical activity and exercise interventions for older adults with $\mathrm{AD}$.

\section{Methods}

\section{Search strategy}

A systematic literature search was performed for eligible studies published before November 1st 2018 on three international databases (PubMed, Cochrane Library, and Embase) and two Chinese databases (Wanfang Data, China National Knowledge Infrastructure). Key words as "exercise" OR "physical activity" OR "physical activities" OR "physical training" AND "Alzheimer Disease" OR "Alzheimer's Disease" OR "Alzheimer-Type Dementia" were searched. 5450 studies were retrieved in this search, which were supplemented by manual searches through reference lists of published reports. Titles and abstracts from a final total of 5462 studies were then reviewed for further inclusion. All analyses were based on published studies, therefore no ethical approval and patient consent are required.

\section{Criteria for inclusion}

Studies were included that met the following inclusion criteria:(1) included subjects diagnosed with AD; (2) involved an exercise-only intervention in the experimental group under the guarantee of basically medical care; (3) included a non-diet, non-exercise control group under the guarantee of basically medical care; (4) pre- and post-intervention cognitive function measurements were reported with means, standard deviations (SDs) of MiniMental State Examination(MMSE) for both exercise and control groups; (5) written in English or Chinese. Two authors (Jiao Ruixia and Liang Jinghong) independently reviewed full texts of all articles that were considered relevant for inclusion in this review. A third author was consulted in cases of disagreements between these two authors. The study selection process is described in Fig. 1.

\section{Criteria for exclusion}

Studies that clearly did not meet initial criteria were rejected on initial review. Reviews, conference papers, abstracts without available full text and studies written in languages other than English and Chinese were also 


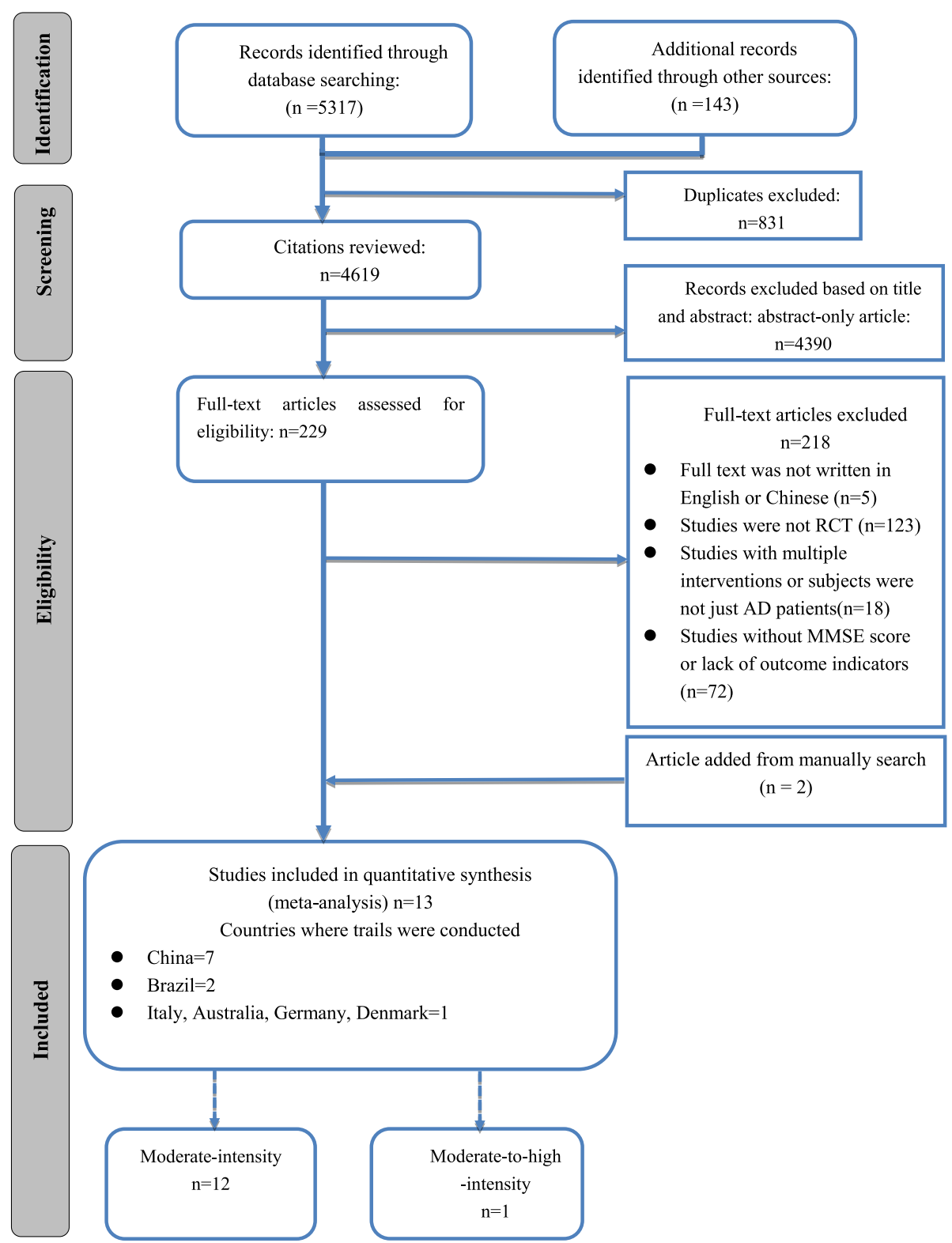

Fig. 1 Literature review flowchart. (AD, Alzheimer's disease; MMSE, Mini-Mental State Examination; RCT, Randomized controlled trial)

excluded. Studies that were qualitative or investigated the effects of exercise on cognitive function in combination with other interventions, such as cognition therapy or cognitive stimulation, were excluded.

\section{Data extraction}

Titles and abstracts of studies identified in the initial search were imported into EndNote for Preliminary screening. After duplicates, studies that are clearly irrelevant to the effects of physical activity and exercise on $\mathrm{AD}$ and animal trails were removed; full texts of potentially relevant papers were imported for further screening. Two authors (RXJ, JHL) performed an initial screening of titles and abstracts independently followed by a screening potential relevant full texts guided by inclusion criteria. A third reviewer (YQW) was consulted for any screening disagreement.

From each study, we collected the following data if available: year of publication, sample size and participants' characteristics (age, number of participants from experimental groups and control group, gender proportion, country, pre- and post-intervention MMSE score, adverse effects and conclusions), intervention characteristics (type, length of whole duration, frequency, time of single session, intensity of intervention). Relevant information was extracted by two reviewers independently onto a standard template. 


\section{Risk of bias}

Studies meeting inclusion criteria were individually scored by two authors (RXJ, JHL) independently according to the Cochrane risk of bias tool, the third author (YQW) would be consulted when disagreement appeared. 7 items regarding risk of bias was assessed: random sequence generation, allocation concealment, blinding of participants and personnel, blinding of outcome assessment, incomplete outcome data, selective outcome reporting, and other sources of bias. The included RCTs were classified as being at low risk, high risk, or unclear risk in the above fields.

\section{Data analysis}

All analyses were conducted using Stata 14.0. Due to heterogeneity between studies, a random-effects model was used for this meta-analysis. Subgroup analyses were conducted to explore the differences among the effects of various types of physical activity and exercise on cognitive performance of subjects with AD.

\section{Meta-analysis}

Random-effects meta-analysis was selected due to the existence of methodological heterogeneity across studies. Standardized mean differences (SMD) were pooled because outcomes were continuous. Heterogeneity across studies was quantified using I squared statistic (variation in SMD attributable to heterogeneity).

\section{Subgroup analysis}

Studies were grouped based on country, time of a single session, intervention time per week, frequency per week and length of whole duration. Intervention effects were estimated within subgroups and compared across subgroups to identify components that modify the intervention effects.

\section{Results}

\section{Study selection}

The original search identified 5462 citations. 229 citations underwent a full-text screen by two independent reviewers (RXJ, JHL), after duplicates, studies irrelevant to $\mathrm{AD}$ and exercise or physical activity, animal trails and reviews were removed. A total of 13 trials were included in the final review (see Additional file 1. A full report of the selection process can be found in the PRISMA diagram in Fig. 1.

\section{Description of studies}

Descriptive characteristics for 13 included studies are presented in Table 1 . Of the 13 trials, seven were based in China [15-21], two in Brazil [22, 23], and one each in Italy [24], Australia [25], Germany [26] and Denmark [27]. In total, the trials included 673 participants, with
200 recruited from one trial [27]. Four studies reported 26 drop-out subjects in total, which leaved 647 subjects. The 13 included studies were published between 2008 and 2017. All studies reported beneficial effects of physical activity on cognition of patients with AD.

Among all included studies, random assignment procedure were reported in 7 studies; Only 5 studies reported a blinding procedure; Analyses accounting for patient drop-out during intervention were presented in 5 studies; All studies described the exercise session duration and reported MMSE scores. Figures 2 and 3 presented the degree of risk of bias for all studies included.

\section{Participants' characteristics}

All included trials contained a sample size of 673 subjects diagnosed with AD. Exercise and control groups were composed of 342 and 331 subjects respectively. A total of 26 subjects dropped out, among which 13 were from experimental groups and the other 13 were from control groups, leaving 647 subjects completing these RCTs. The percentage of female participants was $56.16 \%$ at baseline. Subjects' average age was 73.53 (range from 51 to 89 ) with a mean Mini Mental State Examination score of subjects was 20.09 at baseline (Table 1).

\section{Intervention characteristics}

Table 1 showed that of 13 included studies, only one carried out moderate-to-high intensity intervention, the rest of included studies conducted moderate intensity exercise or physical activity. Aerobic exercise was chosen by 11 studies. All interventions were conducted at an average of $40 \mathrm{~min}$ a session ranging from 30 to $60 \mathrm{~min}$. Interventions were conducted at least one hour a week. Length of whole duration ranges from 12 to 24 weeks, mean intervention duration was 16.92 weeks. Only one study reported follow-up MMSE score 3 months after the intervention. One study reported adverse effect and one study did intention-to-treat (ITT) analysis.

\section{Meta-analysis \\ Primary outcome}

Intervention groups showed a statistically significant improvement in cognition of included subjects measured by the MMSE score $(\mathrm{SMD}=1.12 \mathrm{CI}: 0.66 \sim 1.59$ I-squared = $85.0 \% p=0.000$ ) compared to the control groups (Fig. 4). After exclusion of 6 studies [16-18, 20,21,27] based on funnel plots, we found positive overall random effects of physical activity or exercise interventions on cognitive function $(\mathrm{SMD}=1.94 \mathrm{CI}: 1.59 \sim 2.29$ I-squared $=0.0 \% p=$ 0.461) (Fig. 5). To explain the heterogeneity between 13 studies and find modifiable factors of physical activity and exercise, we did five further subgroup analyses. 


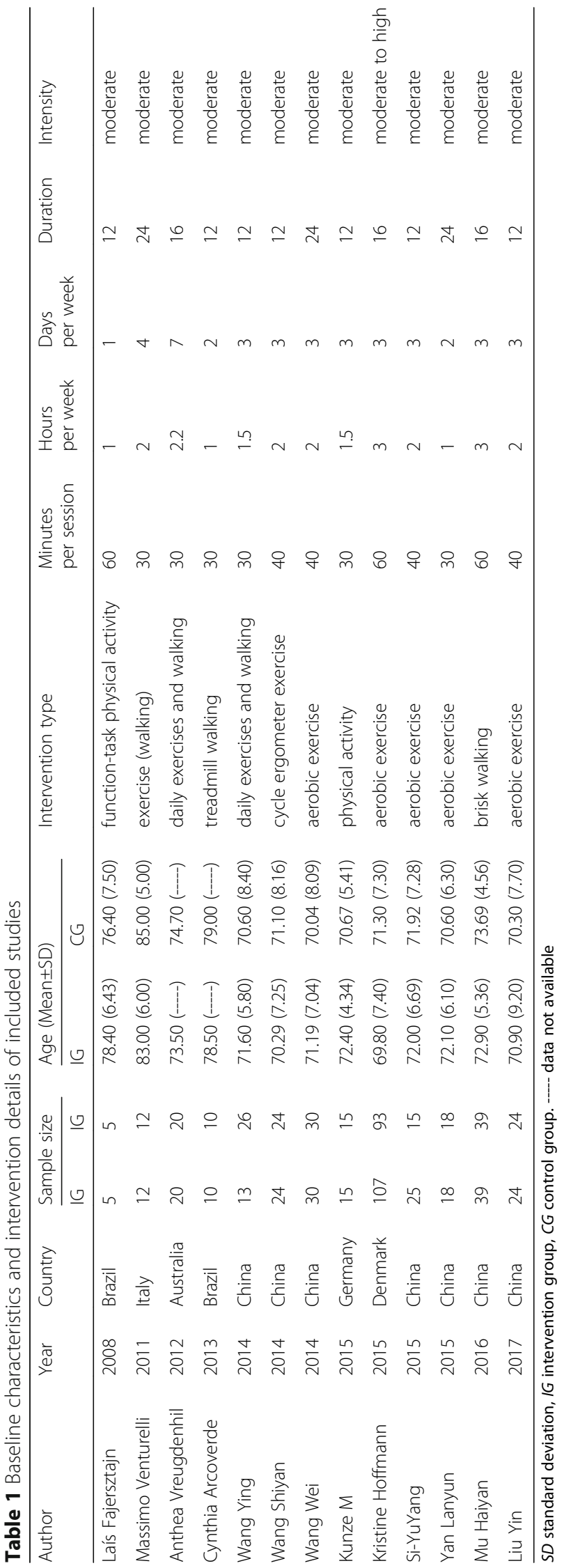




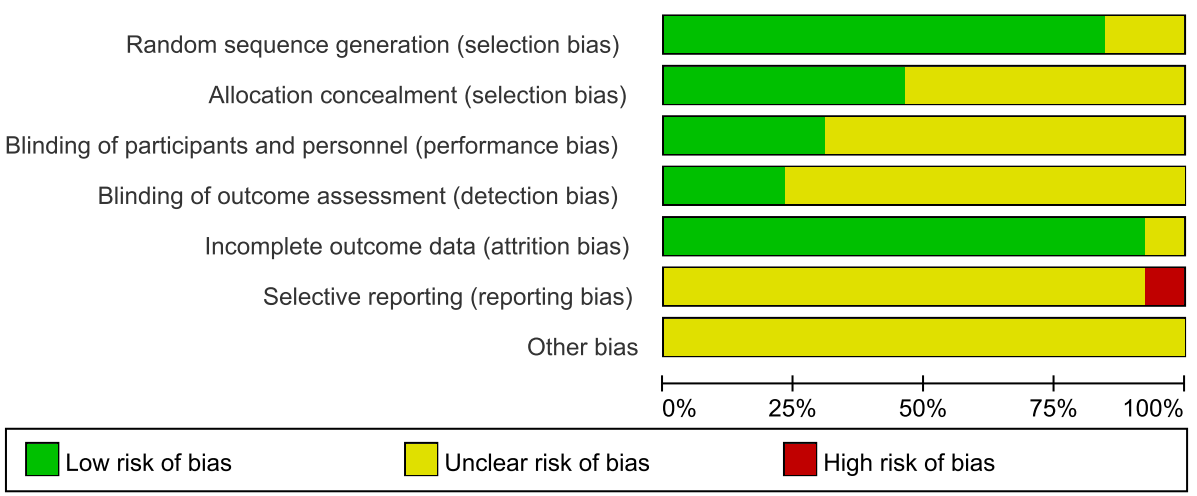

Fig. 2 Assessment for risk of bias

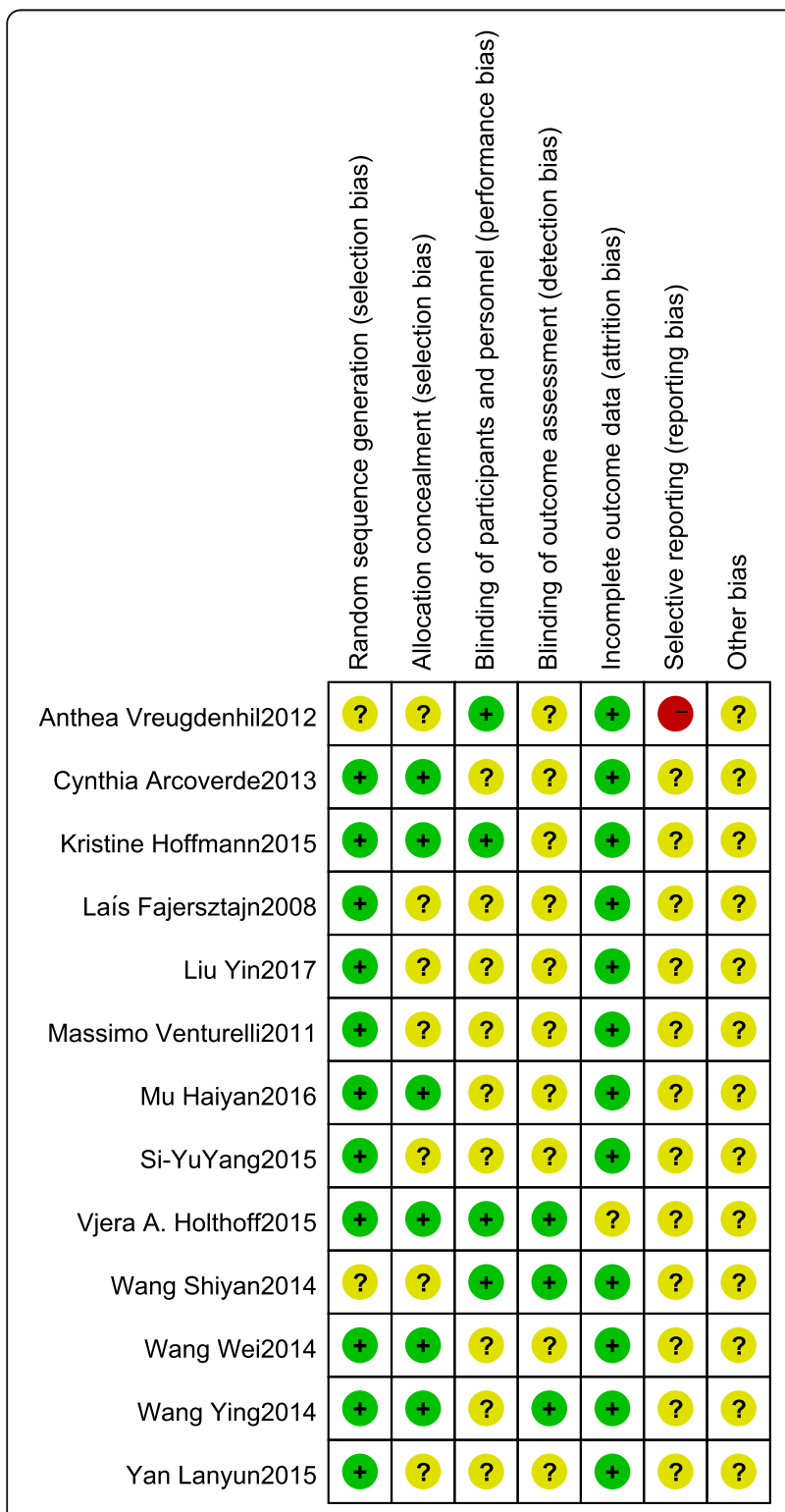

Fig. 3 Assessment for the risk of bias in included studies

\section{Secondary outcome}

\section{Subgroup analysis}

1st subgroup categorized by country

Group 1 (interventions conducted in countries other than China) (SMD = 1.77 CI:0.61 2.93 I-squared $=$ $91.6 \%, p=0.000)$ showed a greater positive effect on cognition compared to group 2 (interventions conducted in China) (SMD $=0.70 \mathrm{CI}: 0.28 \sim 1.12 \mathrm{I}$ squared $=69.7 \%, p=0.000$ ). (Fig. 6). Although it is hard to draw a final conclusion on this due to weak evidence, the tendency is noteworthy.

2nd subgroup categorized by minutes of intervention per session

Group 1 (interventions conducted up to $30 \mathrm{~min}$ per time) (SMD $=1.92$ CI:1.55 2.30 I-squared $=8.3 \%$, $p=0.363)$ showed greater effectiveness for improving cognition of patients compared to group 2 (those conducted more than $30 \mathrm{~min}$ per time) ( $\mathrm{SMD}=0.34$ CI:0.08 0.61 I-squared $=39.4 \%, p=0.129$ ) (Fig. 7).

3rd subgroup categorized by hours of intervention per week Interventions conducted up to $2 \mathrm{~h}$ per week $(\mathrm{SMD}=1.74$ CI:1.32 2.15 I-squared $=0.0 \%, p=0.659$ ) have a tendency to show greater effectiveness for improving cognition of patients compared to those conducted more than $2 \mathrm{~h}$ per week $(\mathrm{SMD}=0.76$ CI:0.27 1.24 I-squared $=$ $84.0 \%, p=0.000$ ) (Fig. 8).

\section{4th subgroup categorized by frequency of intervention per week}

Interventions conducted less than 3 times including 3 times per week $(\mathrm{SMD}=1.58$ CI:1.01 2.14 I-squared $=$ $0.0 \%, p=0.61$ ) showed greater effect on improving cognition of patients compared to those conducted more than 3 times per week $(\mathrm{SMD}=0.99 \mathrm{CI}$ : $0.49 \sim 1.50$ I-squared $=86.5 \%, p=0.000)$ (Fig. 9). 


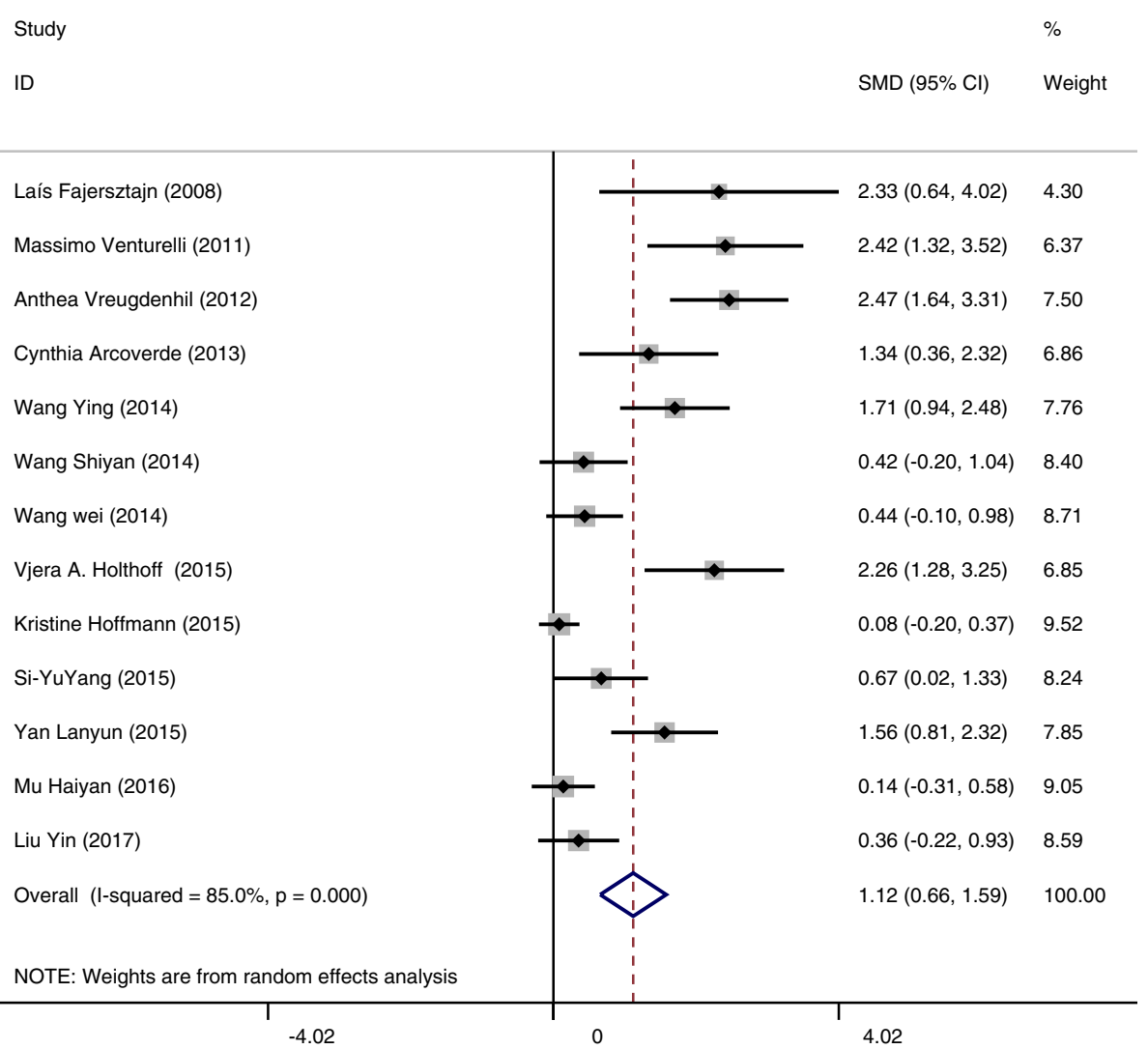

Fig. 4 Effects of physical activity and exercise on the outcome of cognitive function of patients with AD (13 studies)

\section{5th subgroup categorized by length of whole intervention duration}

Interventions conducted more than 16 weeks $(\mathrm{SMD}=$ 1.60 CI:0.62 2.59 I-squared $=83.1 \%, p=0.000$ ) showed greater effect on improving cognition of patients compared to those conducted up to 16 weeks. (SMD $=0.91$ CI:0.40 1.43 I-squared $=83.9 \%, p=0.000$ ) (Fig. 10).

\section{Publication bias}

No obvious publication bias was found.

\section{Discussion}

There are a growing number of studies suggesting that physical activity and exercise have positive effects on cognitive function of the elderly [28-30]. A systematic review [31] showed the relation between exercise and improvement in overall cognitive function as seen by an improvement in scores on the MMSE and the ADASCog. A previous meta-analysis of 29 randomized controlled trials $(n=2049)$ showed that aerobic exercisers exerted positive effects on executive function [32]. Sergio Machado et al [33] suggested that physical exercise was an important neuroprotective modulator, controlling the disease and amplifying brain functions in a significant way. The findings of a review [34] confirmed that in most cases physical activities had a positive effect on the improvement of cognitive decline in $\mathrm{AD}$. In a systematic review, Ruth Stephen et al [35] hold that physical activity is particularly protective against AD. A new review [36] concluded that physical exercise determines positive biological and psychological effects that affect the brain and the cognitive functioning. Tyndall, A. V. et al [37] proposed physical activity, particularly aerobic exercise, through a number of mechanisms has positive effects on blood biomarkers, physiology, and psychological factors associated with cognitive functioning. The positive effect of physical activity and exercise on cognition has been proved by aforementioned studies, which is in consistent with our findings. All improvement in scores on the MMSE from intervention groups of included studies showed that patients in intervention groups with a period of physical activity or exercise had an improved cognition performance while those in control groups did not. But it is noteworthy that there is subtle difference between various trails conducted in China and other countries, for which the reason is left to be discovered. In this term, there should be more RCTs involving subjects from different political, cultural, geographic, religious and dietary backgrounds to explore if there are any cofactors in these above respects 


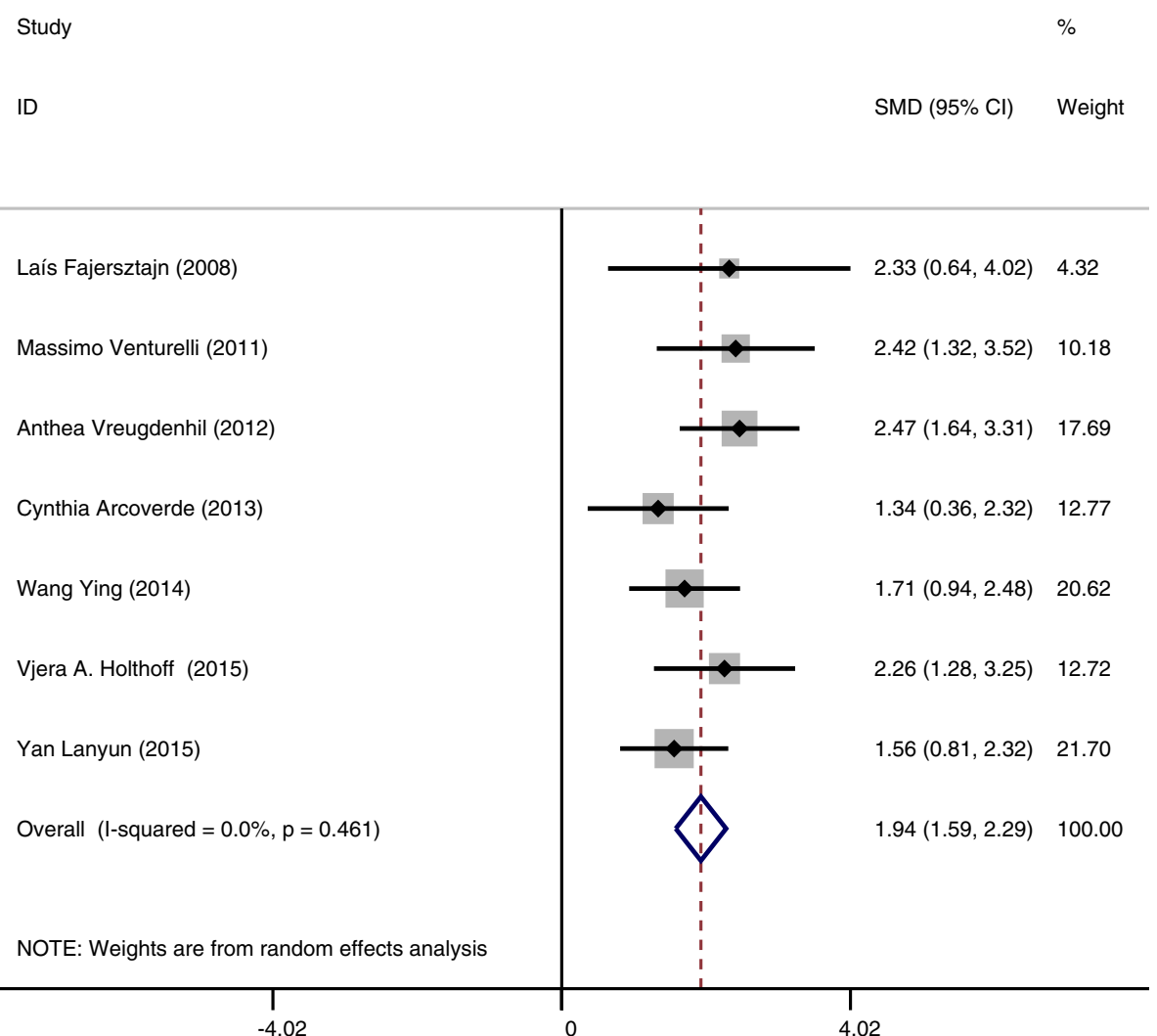

Fig. 5 Effects of physical activity and exercise on the outcome of cognitive function of patients with AD (7 studies)

affecting the cognition of elderly with AD together with physical activity and exercise.

Even though there is robust evidence supporting the effects of physical activity and exercise on cognition of patients with $\mathrm{AD}$, the mechanism is still left to be discovered. The mechanisms Patrick J. Gallaway et al. [34] proposed several potential mechanisms as physical activity can increase blood flow to the brain, improve sleep quality, improve cardiovascular and metabolic health, prevent and treat depression. However, the potential confounding effects of the aged brain cognitive reserve can not be neglected. Francesca Gelfo [38] et al. described animal studies focusing on neuroanatomical and molecular effects of environmental enrichment to address the biological bases of the experience-dependent "brain reserve", which is to say that enriched environment is beneficial for brain cognitive reserve. A population-based study [39] reported that environments that offer physical, cognitive or social interaction stimuli may be conductive in increasing cognitive reserve or compensate for damaging processes. A study conducted in German [40] showed that increased left frontal cortex-connectivity was associated with higher reserve in the memory domain. Nicolai Franzmeier et al [41] then reinforced this theory by proposing that interactions between the left frontal cortex and specific functional networks supported reserve in $\mathrm{AD}$. In a review article [42], the mechanisms underlying the neuroprotective effect of brain reserve was also discussed. Based on these studies, in addition to the benefit of physical activity and exercise on cognition, it should also be considered that the contribution brought by brain reserve to improve the cognition performance of patients with AD. Hence future studies on the effects of physical activity and exercise need to consider the confounding effect of brain reserve to find out to what exact extent physical activity and exercise can improve cognition performance of patients with $\mathrm{AD}$.

It is well established that physical activity and exercise are beneficial for AD patients' cognition, yet few studies proposed a specific way of exercise or physical activity that could exert a positive effect on AD. Öhman H [43] et al. found the outcomes of cognitive tests did not correlate with the number of sessions attended. Shane P. Cass [44] et al. stated that it is not clear if the relationship is dose dependent. Yet on the contrary Yu Fang stated that different ways of exercise or physical activity may confer different effects [45]. Roach K E et al. concluded that specificity of training is one important training parameter in designing successful exercise 


Study
ID

Fig. 6 Subgroup analysis on the outcome of cognitive function categorized by country (Group 1 interventions in countries other than China; Group 2 interventions in China)

programs [46]. BM Brown [47] held that significant cognitive benefits required a threshold level of exercise or physical activity. Our study indicated that adopting appropriate exercise or physical activity is of vital importance to improve cognition of patients with $\mathrm{AD}$.

There has also been a lot of controversy on the doseresponse relationship between physical activity or exercise and cognition. Several institutions and studies recommended different versions of quantity and quality of exercise for preventing cognition decline.

The American College of Sports Medicine and the American Heart Association [48] suggest that to promote and maintain health, older adults need moderateintensity aerobic physical activity for a minimum of 30 min on five days each week or vigorous-intensity aerobic activity for a minimum of $20 \mathrm{~min}$ on three days each week. According to the World Health Organization [49], people over 65 years old fully functional in cognition should adopt a weekly minimum of $150 \mathrm{~min}$ of moderate-intensity aerobic or $75 \mathrm{~min}$ of vigorousintensity aerobic activity with additional musclestrengthening exercises to reduce the risk of cognitive decline, which also suits individuals with neurodegenerative disease. Neal D. Barnard et al. [50] state that adopting 40 min of brisk walking 3 times per week as a routine activity can improve cognitive function, however in our study 30 min physical activity or exercise appears to be more effective. Although with the limitation of comparison among different types of physical activity and exercise, we could not conclude on which combination is best, but from the whole picture we could see trails conducted $30 \mathrm{~min}$ do have a better effects on cognition function compared to those conducted more than $30 \mathrm{~min}$. Lucia A [51] et al. hold that it might be possible to prevent the risk of cognitive deterioration by promoting $\geq 30 \mathrm{~min}$ on five days a week of moderate-intensity aerobic PA, or vigorous-intensity aerobic PA for a minimum of 20 min on three days each week.

After comparing 12 trials conducting moderateintensity exercise or physical activity intervention on patients with $\mathrm{AD}$, we found that the concomitant effects on cognition functions of high frequency interventions was not greater than that of low frequency interventions, which is in consistency with what Groot C [52] et al. found that positive effect on cognition was smaller in high frequency interventions compared to low frequency interventions. Our study showed that interventions conducted up to $30 \mathrm{~min}$ per time have greater effectiveness for improving cognition of patients compared to those conducted more than $30 \mathrm{~min}$ per time; Interventions conducted up to $2 \mathrm{~h}$ per week have a slight tendency to show greater effectiveness for improving cognition of 


Study
ID

Fig. 7 Subgroup analysis on the outcome of cognitive function categorized by minutes of intervention per session (Group 1 up to 30 min per session; Group 2 more than 30 min per session)

patients compared to those conducted more than $2 \mathrm{~h}$ per week; However due to the obvious heterogeneity between interventions conducted more than two hours a week and the limited number of interventions conducted up to $2 \mathrm{~h}$ a week, the evidence from the subgroup analysis of intervention hours per week is not strong enough to determine which subgroup is better. Interventions conducted less than 3 times including 3 times per week showed greater effect on improving cognition of patients compared to those conducted more than 3 times per week. Although Kramer et al [53], Van Gelder et al [54] did quantified studies finding that the minimal time of physical activity or exercise that could benefit cognition is $1.5 \mathrm{~h}$ per week for elderly. It is not clear if this could apply to patients with AD, we still need to find out the low limit time of single session that could exert positive effects on cognition of older adults with $\mathrm{AD}$ in that some of them are too weak to be physically active.

Although we could not draw a conclusion on whether high-intensity or moderate-intensity is better due to limited studies include, there is a noteworthy question need paying attention to that studies are not in consistent in the term of "intensity". While some studies defined intensity in percentage of heart rate reserve $[47,55,56]$, some did with maximal oxygen consumption (VO2peak)
[57, 58] or percentage of maximal heart rate [59], a study even defined intensity as intervention minutes per session [60]. We deem it is urgent to set a standard system of definition involved in physical activity and exercise aimed at improving cognition of older adults with AD.

As for frequency, we think it is important to categorize the term frequency into the following three specific aspects (1). How long of intervention in a single session; (2). How long of intervention per week; (3) how many times intervention per week. In that results of our analysis show that of the above aspects may influence effects of exercise on cognitive function. But the best combination is yield to be discovered. By clarifying the term frequency, it is more likely to find a better intervention method to achieve goals in an accurate way.

In regard to the length of the whole duration, Ashwini K. Rao [60] concluded in a meta-analysis that longer duration of intervention was not associated with better outcomes. Rather, larger effect sizes were seen in studies with shorter and midrange intervention duration. While in our study, interventions conducted more than 16 weeks did have a better effect than those less than 16 weeks. But due to limited studies, the evidence is weak.

According to our results of this study, we would make a modest recommendation that patients with $\mathrm{AD}$ should 


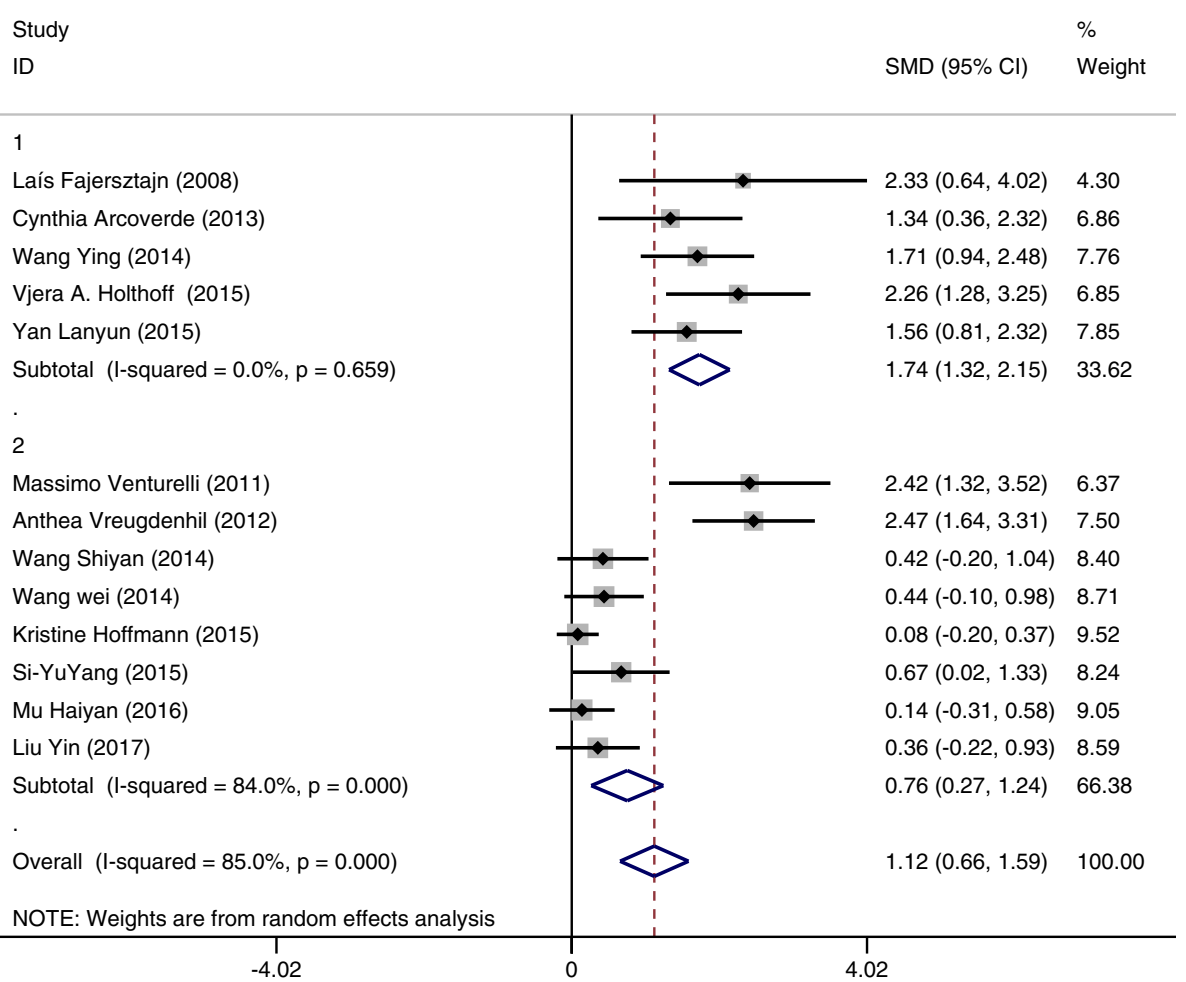

Fig. 8 Subgroup analysis on the outcome of cognitive function categorized by hours of intervention per week (Group 1 up to two hours per week; Group 2 more than $2 \mathrm{~h}$ per week)

Study

ID$$
1
$$

Laís Fajersztajn (2008)

Cynthia Arcoverde (2013)

Yan Lanyun (2015)

Subtotal (I-squared $=0.0 \%, p=0.610$ )

2

Massimo Venturelli (2011)

Anthea Vreugdenhil (2012)

Wang Ying (2014)

Wang Shiyan (2014)

Wang wei (2014)

Vjera A. Holthoff (2015)

Kristine Hoffmann (2015)

Si-YuYang (2015)

Mu Haiyan (2016)

Liu Yin (2017)

Subtotal (I-squared $=86.5 \%, p=0.000)$

Overall (I-squared $=85.0 \%, p=0.000)$

NOTE: Weights are from random effects analysis

1
-4.02

$\%$

SMD $(95 \% \mathrm{Cl}) \quad$ Weight

$2.33(0.64,4.02) \quad 4.30$

$1.34(0.36,2.32) \quad 6.86$

$1.56(0.81,2.32) \quad 7.85$

$1.58(1.01,2.14) \quad 19.01$

$2.42(1.32,3.52) \quad 6.37$

$2.47(1.64,3.31) \quad 7.50$

$1.71(0.94,2.48) \quad 7.76$

$0.42(-0.20,1.04) \quad 8.40$

$0.44(-0.10,0.98) \quad 8.71$

$2.26(1.28,3.25) \quad 6.85$

$0.08(-0.20,0.37) \quad 9.52$

$0.67(0.02,1.33) \quad 8.24$

$0.14(-0.31,0.58) \quad 9.05$

$0.36(-0.22,0.93) \quad 8.59$

$0.99(0.49,1.50) \quad 80.99$

$1.12(0.66,1.59) \quad 100.00$

Fig. 9 Subgroup analysis on the outcome of cognitive function categorized by frequency of intervention per week (Group 1 up to three times per week; Group 2 more than 3 times per week) 


Study
ID

Fig. 10 Subgroup analysis on the outcome of cognitive function categorized by length of whole intervention duration (Group 1 duration up to 16 weeks; Group 2 duration more than 16 weeks)

do physical activity or exercise up to 3 times a week with 30 min per session. However this recommendation still needs verification by more targeted studies.

\section{Strengths, limitations, and future directions}

The primary strength of our meta-analysis lies in its multiple-subgroup design, which provides better quantification of the associations between specified amounts of physical activity or exercise and relative effects on AD. Another strength is that only RCT studies were included. The included studies were conducted in both developed countries like Germany and Denmark and developing countries like China and Brazil, therefore these findings also apply to both kinds of countries. Last, we focused on a single type of dementia (AD), whereas past metaanalyses have typically included trials of subjects with multiple types of dementia [61-63] or subjects with a diagnosis only of cognitive impairment.

There are several limitations that might affect the reporting of our results. First, the review was limited to two languages English and Chinese. Hence, studies published in other languages were not captured, which may result in a limited number of include studies. Second, the types of physical activity or exercise are quite different, and the way researchers conducting interventions are not in identically standard criteria, so there is heterogeneity between included studies. Third, limited studies led to the failure to identify the optimal type to improve cognition of $\mathrm{AD}$ patients. And we failed to conduct the intensity analysis due to the restriction of data. Fifth, we cannot conclude on how long the effects will last in the reference group due to data restrictions. A majority of researchers seemed to have neglected this important issue, which warrants more attention in the future study that follow-up data is of vital importance. Sixth, we cannot exclude the potentially effects caused by some confounders. Due to the above limitations, more high-quality RCTS are needed to confirm the effects of physical activity and exercise on improving cognition of patients with $\mathrm{AD}$ in the future.

Standard criteria need to be established for future studies in several aspects. (1) Description of characteristics. Of all the reviewed RCT studies rendered ineligible, a majority $(n=22)$ we reviewed were deemed ineligible due to the lack of MMSE or scores not being presented in the way of Mean \pm Standard Deviation; (2) Intervention design. Standard definition of terms as intensity, frequency, duration need to be settled to describe specific aspects of physical activity and exercise to find a more likely better intervention method to achieve goals in an accurate way. On the other hand, follow-up effects need to be in record to understand how long the effects 
could last. More RCTs with large sample are needed to verify the effects of physical activity and exercise on cognition of patients with $\mathrm{AD}$ around the whole world.

\section{Conclusion}

Considering that there is no direct treatment for $\mathrm{AD}$, it is urgent to find a way to delay its progress. Physical activity and exercise have been shown to aid in achieving this goal in a cost effective and sustainable manner. The results from this study shows that physical activity and exercise can achieve improvement in cognition of elderly with $\mathrm{AD}$ and high frequency interventions are not necessarily characterized with greater effects on cognition functions compared to low frequency interventions, we still need to find out the threshold of physical activity and exercise that could exert a positive effect on cognition performance of patients with AD. More highquality randomized controlled trials with less methodological issues and noted heterogeneity are required to back up our present findings.

\section{Additional file}

Additional file 1: PRISMA 2009 Checklist. (PDF 217 kb)

\section{Abbreviations}

AD: Alzheimer's disease; CG: Control group; IG: Intervention group; MMSE: Mini-Mental State Examination; RCT: Randomized controlled trial; SD: Standard deviation; SMD: Standard Mean Difference

\section{Acknowledgements}

The authors would like to gratefully thank Hongbo Zhang and Lei Hang for their help.

\section{Authors' contributions}

RXJ conducted the literature search, screened and extracted data for the meta-analysis, prepared extracted data for the procedures, and had primary responsibility in writing this article. JHL performed statistical analysis and interpretation of data. YQW made contribution in the process of discussion and editing. YX critically revised the draft manuscript. All authors read and approved the final manuscript.

\section{Funding}

No specific funding was received for this study.

\section{Availability of data and materials}

All data generated or analysed in this study are included in this published article (and its Additional file 1).

Ethics approval and consent to participate

Not applicable.

\section{Consent for publication}

Not applicable.

\section{Competing interests}

The authors declare that they have no competing interests.
Received: 14 June 2018 Accepted: 30 May 2019

Published online: 02 July 2019

\section{References}

1. Herrera AC, Prince M, Knapp M, et al. Improving healthcare for people with dementia. Coverage, quality and costs now and in the future. World Alzheimer Report. 2016;2016.

2. Gaugler J, James B, Johnson T, et al. 2016 Alzheimer's disease facts and figures. Alzheimers Dement. 2016;12(4):459-509.

3. Burns A, Byrne EJ, Maurer K. Alzheimer's disease. BMJ (Clinical research ed). 2009;338(7692):467-71.

4. Querfurth HW, Laferla FM. Alzheimer's disease. N Engl J Med. 2010;362(4):329.

5. Todd S, Barr S, Roberts M, et al. Survival in dementia and predictors of mortality: a review. Int J Geriatr Psychiatry. 2013;28(11):1109-24.

6. Vanhanen $M$, Kivipelto $M$, Koivisto $K$, et al. APOE-epsilon4 is associated with weight loss in women with AD: a population-based study. Neurology. 2001:56(5):655-9.

7. Cedazomínguez A. Apolipoprotein E and Alzheimer's disease: molecular mechanisms and therapeutic opportunities. J Cell Mol Med. 2007;11(6): 1227-38.

8. Rolland Y, Kan GAV, Vellas B. Physical activity and Alzheimer's disease: from prevention to therapeutic perspectives. J Am Med Dir Assoc. 2008:9(6):390-405.

9. Fratiglioni L, Paillard-Borg S, Winblad B, Fratiglioni L, Paillard-Borg S, Winblad B. An active and socially integrated lifestyle in late life might protect against dementia. Lancet Neurol. 3, 343-353. Lancet Neurol. 2004;3(6):343-53.

10. Hertzog C, Kramer AF, Wilson RS, et al. Enrichment effects on adult cognitive development: can the functional capacity of older adults be preserved and enhanced? Psychol Sci Public Interest. 2008:9(1):1-65.

11. Kramer AF, Bherer L, Colcombe SJ, et al. Environmental influences on cognitive and brain plasticity during aging. J Gerontol. 2004;59(9):M940.

12. Lautenschlager NT, Cox KL, Flicker $\mathrm{L}$, et al. Effect of physical activity on cognitive function in older adults at risk for Alzheimer disease: A randomized trial. JAMA- J Am Med Assoc. 2008;300(9):1027-37. https://doi. org/10.1001/jama.300.9.1027 [published Online First: Epub Date].

13. Kramer AF, Erickson Kl, Colcombe SJ. Exercise, cognition, and the aging brain. J Appl Physiol. 2006;101(4):1237-42

14. Heyn P, Abreu BC, Ottenbacher KJ. The effects of exercise training on elderly persons with cognitive impairment and dementia: a meta-analysis. Arch Phys Med Rehabil. 2004:85(10):1694-704.

15. Wang Y, Shen FF, Zhu Y, et al. Clinical Effects of Aerobic Exercises Training with Moderate and High Intensity in Alzheimer's Disease Treatment. Chin J Clin Oncol Neurosci. 2014;22(5):504-9

16. Wang SY, Zhu Y, Zhang Q. The effect of aerobic exercise of middle intensity on cognitive and motor functions of patients with Alzheimer's disease. Chin J Rehabil Med. 2014;36(10):765-8.

17. Wang Wei ZY, Yang Si Y. Effects of aerobic exercise on cognition function and activity of daily life of patients with Alzheimer's disease. Chinese. J Rehabil Med. 2014;29(12):1151-5.

18. Yang SY, Shan $\mathrm{CL}$, Qing $\mathrm{H}$, et al. The effects of aerobic exercise on cognitive function of Alzheimer's disease patients. CNS Neurol Disord Drug Targets. 2015;14(10):1292-7.

19. Yan L, Wang W, Shen F. A clinical research of aerobic exercises with different training time in the treatment of mild to moderate Alzheimer's disease. Chin J Rehabil Med. 2015;30(8):771-6.

20. Yin LTW, Yi Z. Aerobic exercise improves cognitive function in patients with Alzheimer's disease. Chin J Rehabil. 2017;32(5):386-9.

21. Yan MHHL, Hui HZ. Effect of aerobic exercise on abilities of daily life, cognitive function and psychological symptoms in patients with mild to moderate Alzheimer's disease. Chin J Multiple Organ Dis Elder. 2016:15(6):451-4

22. Fajersztajn $L$, Cordeiro RC, Andreoni $\mathrm{S}$, et al. Effects of functional physical activity on the maintenance of motor function in Alzheimer's disease. Dement Neuropsychol. 2008;2(3):233-40.

23. Arcoverde $\mathrm{C}$, Deslandes $\mathrm{A}$, Moraes $\mathrm{H}$, et al. Treadmill training as an augmentation treatment for Alzheimer's disease: a pilot randomized controlled study. Arq Neuropsiquiatr. 2014;72(3):190-6.

24. Venturelli M, Scarsini R, Schena F. Six-month walking program changes cognitive and ADL performance in patients with Alzheimer. Am J Alzheimers Dis Other Dement. 2011;26(5):381. 
25. Vreugdenhil A, Cannell J, Davies A, et al. A community-based exercise programme to improve functional ability in people with Alzheimer's disease: a randomized controlled trial. Scand J Caring Sci. 2012;26(1):12-9.

26. Kunze $M$, Voss $P$, Büttner $H H$, et al. Effects of physical activity training in patients with Alzheimer's dementia: results of a pilot RCT study. PLoS One. 2015;10(4):e0121478

27. Hoffmann K, Sobol NA, Frederiksen KS, et al. Moderate-to-high intensity physical exercise in patients with Alzheimer's disease: a randomized controlled trial. J Alzheimers Dis Jad. 2015;11(7):P324-P25.

28. Chapman SB, Aslan S, Spence JS, et al. Shorter term aerobic exercise improves brain, cognition, and cardiovascular fitness in aging. Front Aging Neurosci. 2013;5(75):75.

29. Farina N, Rusted J, Tabet N. The effect of exercise interventions on cognitive outcome in Alzheimer's disease: a systematic review. Int Psychogeriatr. 2014;26(1):9-18

30. Yu F, Kolanowski AM, Strumpf NE, et al. Improving cognition and function through exercise intervention in Alzheimer's disease. J Nurs Scholarsh. 2006;38(4):358-65.

31. Rao AK, Chou A, Bursley B, et al. Systematic review of the effects of exercise on activities of daily living in people with Alzheimer's disease. The American journal of occupational therapy : official publication of the American occupational. Therapy Association. 2014;68(1):50-6.

32. Smith PJ, Blumenthal JA, Hoffman BM, et al. Aerobic exercise and neurocognitive performance: a meta-analytic review of randomized controlled trials. Psychosom Med. 2010;72(3):239-52.

33. Machado S, Filho A, Wilbert M, et al. Physical exercise as stabilizer for Alzheimer's disease cognitive decline: current status. Clin Pract Epidemiol Mental Health Cp Emh. 2017;13(1):181-4.

34. Gallaway PJ, Miyake H, Buchowski MS, et al. Physical activity: a viable way to reduce the risks of mild cognitive impairment, Alzheimer's disease, and vascular dementia in older adults. Brain Sci. 2017;7(2):22.

35. Stephen R, Hongisto K, Solomon A, et al. Physical activity and Alzheimer's disease: a systematic review. The journals of gerontology Series A. Biol Sci Med Sci. 2017;72(6):733-9.

36. Mandolesi L, Polverino A, Montuori S, et al. Effects of physical exercise on cognitive functioning and wellbeing: biological and psychological benefits. Front Psychol 2018;9:509.

37. Tyndall AV, Clark CM, Anderson TJ, et al. Protective effects of exercise on cognition and brain health in older adults. Exerc Sport Sci Rev. 2018;46(4): 215-23. https://doi.org/10.1249/jes.0000000000000161 [published Online First: Epub Date]|.

38. Gelfo F, Mandolesi L, Serra L, et al. The neuroprotective effects of experience on cognitive functions: evidence from animal studies on the neurobiological bases of brain reserve. Neuroscience. 2018;370:218-35. https://doi.org/10.1016/j.neuroscience.2017.07.065 published Online First: Epub Date.

39. Blasko I, Jungwirth S, Kemmler G, et al. Leisure time activities and cognitive functioning in middle European population-based study. Eur Geriatr Med. 2014;5(3):200-7.

40. Franzmeier N, Göttler J, Grimmer T. Resting-state connectivity of the left frontal cortex to the default mode and dorsal attention network supports Reserve in Mild Cognitive Impairment. Front Aging Neurosci. 2017;9:264.

41. Franzmeier N, Hartmann JC, Taylor ANW, et al. Left frontal hub connectivity during memory performance supports Reserve in Aging and Mild Cognitive Impairment. J Alzheimers Dis. 2017:59(4):1381-92.

42. Freret $T$, Gaudreau P, Schumann-Bard $P$, et al. Mechanisms underlying the neuroprotective effect of brain reserve against late life depression. J Neural Transm. 2015;122(1):55-61.

43. H Ö, Savikko N, Strandberg TE, et al. Effects of exercise on cognition: the Finnish Alzheimer disease exercise trial: a randomized, controlled trial. J Am Geriatr Soc. 2016;64(4):731-8.

44. Cass SP. Alzheimer's disease and exercise: a literature review. Curr Sports Med Rep. 2017;16(1):19.

45. Yu F. Guiding research and practice: a conceptual model for aerobic exercise training in Alzheimer's disease. Am J Alzheimers Dis Other Dement. 2011;26(3):184

46. Roach KE, Tappen RM, Kirk-Sanchez N, et al. A randomized controlled trial of an activity specific exercise program for individuals with Alzheimer disease in long-term care settings. J Geriatr Phys Ther. 2011;34(2):50.

47. Brown BM, Peiffer JJ, Martins RN. Multiple effects of physical activity on molecular and cognitive signs of brain aging: can exercise slow neurodegeneration and delay Alzheimer's disease? Mol Psychiatry. 2013; 18(8):864-74.

48. Nelson M, W Jack R, Steven N, Blair, Duncan PW, et al. ACSM/AHA recommendations, physical activity and public health in older adults. 2007.

49. Cayon A. PAHO WHO / global recommendations on physical activity for health. 2016.

50. Barnard ND, Bush Al, Ceccarelli A, et al. Dietary and lifestyle guidelines for the prevention of Alzheimer's disease. Neurobiol Aging. 2014;35(Suppl 2): S74-8.

51. Lucia A, Ruiz JR. Exercise is beneficial for patients with Alzheimer's disease: a call for action. Br J Sports Med. 2011:45(6):468-9.

52. Groot C, Hooghiemstra AM, Raijmakers PGHM, et al. The effect of physical activity on cognitive function in patients with dementia: a meta-analysis of randomized control trials. Ageing Res Rev. 2016;25:13-23.

53. Kramer AF, Colcombe SJ, Mcauley E, et al. Enhancing brain and cognitive function of older adults through fitness training. J Mol Neurosci. 2003;20(3): 213-21.

54. van Gelder BM, Tijhuis MA, Kalmijn S, et al. Physical activity in relation to cognitive decline in elderly men: the FINE study. Neurology. 2004;63(12): 2316-21.

55. Hoffmann K, Frederiksen KS, Sobol NA, et al. Preserving cognition, quality of life, physical health and functional ability in Alzheimer's disease: the effect of physical exercise (ADEX trial): rationale and design. Neuroepidemiology. 2013:41(3-4):198-207.

56. Yu F, Bronas UG, Suma K, et al. Effects of aerobic exercise on cognition and hippocampal volume in Alzheimer's disease: study protocol of a randomized controlled trial (the FIT-AD trial). Trials. 2014;15(1):394.

57. Erickson Kl, Prakash RS, Voss MW, et al. Aerobic fitness is associated with hippocampal volume in elderly humans. Hippocampus. 2009;19(10):1030-9.

58. Erickson $\mathrm{Kl}$, Weinstein AM, Sutton BP, et al. Beyond vascularization: aerobic fitness is associated with $\mathrm{N}$-acetylaspartate and working memory. Brain Behav. 2012;2(1):32-41.

59. Yu F, Kolanowski A. Facilitating aerobic exercise training in older adults with Alzheimer's disease. Geriatr Nurs. 2009;30(4):250.

60. Rao AK, Chou A, Bursley B, et al. Systematic review of the effects of exercise on activities of daily living in people with Alzheimer's disease. The American journal of occupational therapy : official publication of the American occupational. Therapy Assoc. 2014;68(1):50-6. https://doi.org/10.5014/ajot. 2014.009035 published Online First: Epub Date.

61. Forbes D, Forbes SC, Blake CM, et al. Exercise programs for people with dementia. The Cochrane database of systematic reviews 2015(4):Cd006489. https://doi.org/10.1002/14651858.CD006489pub4.[published Online First: Epub Date].

62. Zeng $Z$, Deng $Y H$, Shuai $T$, et al. Effect of physical activity training on dementia patients: a systematic review with a meta-analysis. Chin Nurs Res. 2016:3(4):168-75.

63. Hess N, Dieberg G, Mcfarlane J, et al. The effect of exercise intervention on cognitive performance in persons at risk of, or with dementia: a systematic review and meta-analysis. J Qilu Nurs. 2014;3(3).

\section{Publisher's Note}

Springer Nature remains neutral with regard to jurisdictional claims in published maps and institutional affiliations.

Ready to submit your research? Choose BMC and benefit from:

- fast, convenient online submission

- thorough peer review by experienced researchers in your field

- rapid publication on acceptance

- support for research data, including large and complex data types

- gold Open Access which fosters wider collaboration and increased citations

- maximum visibility for your research: over $100 \mathrm{M}$ website views per year

At BMC, research is always in progress.

Learn more biomedcentral.com/submission 